\title{
MEK ablation in MCF-7 cells blocks DNA synthesis induced by serum, but not by estradiol or growth factors
}

\author{
NORMAN R. ESTES II ${ }^{1}$, JAIDEEP V. THOTTASSERY ${ }^{1}$, LOUISE WESTBROOK ${ }^{1}$ and FRANCIS G. KERN ${ }^{2}$ \\ ${ }^{1}$ Biochemistry and Molecular Biology Department, Drug Discovery Division, Southern Research Institute, \\ 2000 Ninth Avenue South, Birmingham, AL 35255; ${ }^{2}$ Lexicon Genetics, Inc., The Woodlands, TX 77381, USA
}

Received May 8, 2006; Accepted July 13, 2006

\begin{abstract}
The role of mitogen-activated protein/extracellular signal-regulated kinase kinase (MEK) signaling in estrogen receptor positive $\left(\mathrm{ER}^{+}\right) \mathrm{MCF}-7$ breast carcinoma cells is not well understood. We depleted MEK by cotransfection of MEK1 and MEK2 siRNA duplexes in a MCF-7 derived line (MCF-7/ lacZ, ML-20) and determined its effect on serum, $17 ß$-estradiol $\left(\mathrm{E}_{2}\right)$, and growth factor induced DNA synthesis. MEK knockdown did not decrease fetal bovine serum-induced DNA synthesis in ML-20 cells although it did inhibit DNA synthesis induced by estrogen-stripped calf serum (CCS) suggesting that MEK activation plays an important role in growth signaling induced by serum components other than estrogen. Consistent with this notion, MEK knockdown only modestly decreased DNA synthesis induced by $\mathrm{E}_{2}$-supplemented CCS medium in ML-20 cells. Similarly, MEK knockdown only caused moderate decreases in DNA synthesis induced by fibroblast growth factor-1 (FGF-1) or heregulin- $\beta 1$ (HRGß1) in this media. Also, there were only minimal effects of MEK knockdown in cells treated with growth factor-supplemented serum-free medium. Although MEK depletion inhibited ERK1/2 phosphorylation induced by CCS in these cells, that induced by growth factor supplemented CCS media was relatively unaffected. Similarly, ERK1/2 phosphorylation induced by growth factorsupplemented serum-free media was also relatively unaffected by MEK depletion. These results suggest that pathways regulating DNA synthesis induced by serum in MCF-7 cells are significantly more dependent on constitutive MEK levels than that induced by $\mathrm{E}_{2}$ or growth factors.
\end{abstract}

\section{Introduction}

Activation of the mitogen-activated protein/extracellular signal-regulated kinase kinase (MEK) pathway has been

Correspondence to: Dr Francis G. Kern, Lexicon Genetics Inc., 8800 Technology Forest Pl., The Woodlands, TX 77381, USA

E-mail: fkern@lexgen.com

Key words: mitogen activated protein kinase, MEK, extracellular signal-regulated kinase, RNA, interference suggested by numerous studies to play a role in proliferation, transformation, migration, angiogenesis and apoptosis (1-5). Constitutive activation of MEK has also been shown to contribute to the development of resistance to antiestrogen therapy in estrogen receptor (ER)-positive breast carcinoma cells (6-8). Many studies have suggested an involvement of MEK/ERK signaling in estrogen or growth factor-mediated cell functions in MCF-7 cells, an ER-positive breast carcinoma line (9-12). Incubation of multiple breast cancer cell lines including MCF-7 with the MEK-specific inhibitor U0126 has been shown to arrest growth of serum starved cells and result in death of all cells within 2-4 days (13). However, clonogenicity studies in which MCF-7 cells were treated with another potent MEK-specific inhibitor, PD184352, suggested that these cells were largely refractory to the effects of MEK inhibition in $20 \%$ fetal bovine serum (FBS)-containing medium (14). This suggests that MEK signaling is less critical for proliferation of MCF-7 cells in the presence of FBS. Since FBS contains the estrogens $17 ß$-estradiol $\left(\mathrm{E}_{2}\right)$, estriol, and estrone, and since MCF-7 cells are primarily dependent on the estrogen component of serum for proliferation, the dependence on MEK for $\mathrm{E}_{2}$-induced signaling and proliferation is an important issue.

Several studies show that $\mathrm{E}_{2}$ is capable of inducing ERK1/2 activation in MCF-7 cells (15-18). Lobenhofer et al showed that the MEK inhibitors PD98059 and U0126 were able to reduce the number of MCF-7 cells undergoing $E_{2}$ induced DNA synthesis (19). Similarly, Razandi et al have shown a rapid and sustained increase in ERK1/2 activity and subsequent proliferation of MCF-7 cells in response to $\mathrm{E}_{2}$ and that these effects were inhibited by PD98059 (17). In stark contrast to these studies, others have shown that inhibition of MEK signaling does not significantly affect $\mathrm{E}_{2}$-induced outcomes in MCF-7 cells. For instance, Lewis et al have recently shown that PD98059 had no significant effect on $\mathrm{E}_{2}$-induced phosphorylation of activating transcription factor-2 (20). Other studies in which estrogendeprived cells were treated with $\mathrm{E}_{2}$ reported no activation of the MEK/ERK pathway despite the stimulation of entry into cell cycle progression, and treatment of these cells with U0126 was only partially inhibitory on cell cycle progression (21). Similarly, Bonapace et al reported that treatment of MCF-7 cells with $\mathrm{E}_{2}$ allowed the cells to overcome a $\mathrm{G} 1$ block induced by $\mathrm{HMG}-\mathrm{CoA}$ reductase 
inhibitors and that this effect did not require activation of ERK1/2 (22).

In addition to $E_{2}$, certain growth factors also stimulate MCF-7 growth. Our laboratory has shown that signaling mediated by either fibroblast growth factor (FGF)-4, FGF-1, or heregulin- $\beta 1$ (HRGß1) can each provide alternative growth signals to that provided by ER activation in ML-20 (MCF-7/ LacZ) cells, which results in antiestrogen resistant growth (23-27). Studies from our laboratory looking at the role of MEK/ERK signaling in growth factor-induced antiestrogenresistant growth of ML-20 cells using PD98059 and U0126 indicated that FGF-1 and HRGß1-induced ERK1/2 phosphorylation and cell proliferation were resistant to the effects of MEK inhibition unlike that induced by FBS (27). Furthermore, these studies also showed that FGF-1 and HRGß1, but not FBS could stimulate ERK1/2 activation in the presence of U0126, suggesting stimulus-specific variability in the susceptibility of MCF-7 cells to small molecule inhibitors of MEK signaling (27).

In this study, we sought to further clarify the role of MEK on $\mathrm{E}_{2}$ and growth factor-induced MEK/ERK signaling in ML20 cells by assessing the effects of RNAi-mediated MEK knockdown on $\mathrm{E}_{2}$ and growth factor-induced DNA synthesis and MEK/ERK phosphorylation in either the presence or absence of FBS or charcoal-stripped calf serum (CCS). We show that a cotransfection of siRNA duplexes targeting MEK1 and MEK2 is able to effectively knockdown both total and phosphorylated levels of MEK in ML-20 breast cancer cells. We show that in this cell line, this knockdown inhibits basal levels of DNA synthesis in cells maintained in CCS containing medium but not FBS containing medium. MEK knockdown only moderately inhibits BrdU incorporation induced by $\mathrm{E}_{2}$ or the growth factors FGF-1 and HRGß1 in CCS containing medium and did not affect IGF-1-induced DNA synthesis in this medium. The moderate effects of MEK knockdown on BrdU incorporation induced by FGF-1 or HRGß1 appear at least partially related to serum components as these effects are reduced in SFM. Knockdown of MEK did not affect ERK1/2 phosphorylation induced by these growth factors although basal levels of ERK phosphorylation in CCScontaining medium were diminished in cells cotransfected with MEK1 and MEK2 siRNAs compared to control transfected cells.

\section{Materials and methods}

Chemicals, growth factors and antibodies. Recombinant human IGF-1 and FGF-1 were obtained from Promega (Madison, WI), and recombinant human HRGß1 (residues 176-246 corresponding to the EGF-like domain) was from R\&D Systems (Minneapolis, MN). 17ß-Estradiol ( $\left.E_{2}\right)$ was obtained from Sigma (St. Louis, MO). Phenol Red Free (PRF) and phenol red containing improved modified Eagle's medium (IMEM) were obtained from Mediatech, Inc., (Herndon, VA), and fetal bovine serum (FBS) was obtained from Invitrogen (Carlsbad, CA). In some experiments CCS was used to strip cells of endogenous estrogens. Calf serum was treated with dextran-coated activated charcoal as described before to obtain CCS $(23,28)$. Anti-phospho ERK1/2, anti-ERK1/2, antiphospho MEK, and anti-MEK were obtained from Cell
Signaling Technology (Beverly, MA). Monoclonal anti-actin antibody was obtained from Sigma.

Cell lines, cell culture, and siRNA. ML-20 is a clonally isolated estrogen-responsive line derived from MCF-7 breast adenocarcinoma cells in our laboratory that has a stably transfected LacZ gene and has been described previously $(24,25,28)$. Cells were maintained in IMEM containing $10 \%$ FBS and incubated at $37^{\circ} \mathrm{C}$ in a humidified atmosphere with $5 \% \mathrm{CO}_{2}$. Small interfering RNA (siRNA) sequences targeting either MEK1 or MEK2 have been described previously (29). These and control siRNA duplexes ( siCONTROL $^{\circledR}$ ) with no known homology to rat, mouse, or human genes were obtained from Dharmacon, Inc. (Lafayette, CO).

siRNA transfections. For both BrdU and Western assays, cells were plated at $4 \times 10^{5}$ cells in $60-\mathrm{mm}$ dishes in $5 \%$ FBScontaining media. Cells were cotransfected with siRNAs targeting MEK1 and MEK2 using DharmaFECT ${ }^{\text {TM }} 2$ transfection reagent (Dharmacon, Inc.) 24-h post plating according to the manufacturer's guidelines in Opti-MEM reduced serum media (Invitrogen) at a concentration of $20 \mathrm{nM}$ each to a final total siRNA concentration of $40 \mathrm{nM}$. Control transfected cells were transfected to a final concentration of $40 \mathrm{nM}$.

Immunoblot analyses. ML-20 cells were transfected with siRNAs as described above and $24 \mathrm{~h}$ later the medium was replaced with $5 \% \mathrm{CCS}$, or with $5 \% \mathrm{CCS}$ containing either $\mathrm{E}_{2}$ at a final concentration of $10^{-8} \mathrm{M}$ for the $\mathrm{E}_{2}$ induction studies, or with 5\% CCS containing IGF-1 (10 ng/ml), FGF-1 (20 ng/ $\mathrm{ml})$, or HRGß1 (50 $\mathrm{ng} / \mathrm{ml})$ for the growth factor induction studies. Similar experiments were carried out in serum-free medium. All treatments were for $15 \mathrm{~min}$. Subsequently, cells were lysed using $200 \mu \mathrm{l}$ cell lysis buffer (Cell Signaling Technology) supplemented with $1 \mathrm{mM}$ phenylmethylsulfonyl fluoride. Cell lysate $(35 \mu \mathrm{g})$ was run on Criterion precast $10 \%$ SDS-PAGE gels (Bio-Rad, Hercules, CA). Proteins were transferred onto Protran filters (Bio-Rad) by electroblotting. The blots were then probed with antibodies against the following: phospho-ERK p44/42, total-ERK p44/42, phosphoMEK $1 / 2$, total-MEK1/2, or actin. Blots were initially probed for either phospho-ERK or total MEK, and then stripped with Restore stripping solution (Pierce, Rockford, IL) and reprobed with antibodies against total ERK, phospho-MEK, or actin. Enhanced chemiluminescence with the Supersignal kit (Pierce) was used to detect bands.

BrdU incorporation assay. Cells were plated in 60-mm dishes and transfected as described above. Twenty-four hours after transfection the media was removed and replaced with 3 changes of $5 \%$ CCS over $8 \mathrm{~h}$ followed by plain PRF-IMEM overnight. For BrdU assays, the serum-free PRF-IMEM was replaced with either $5 \% \mathrm{CCS}$ or $5 \%$ FBS for the serum induction assays, or with $5 \% \mathrm{CCS}$ or SFM containing $\mathrm{E}_{2}$ at a final concentration of $10^{-8} \mathrm{M}$ for the $\mathrm{E}_{2}$ induction studies, or with $5 \%$ CCS or SFM containing IGF-1 (10 ng/ml), FGF-1 (20 $\mathrm{ng} / \mathrm{ml})$, or HRGß1 (50 ng/ml) for the growth factor induction studies. A preliminary time-course study was performed which determined that the optimal duration of treatment with the conditioned media was $24 \mathrm{~h}$ prior to 
A)

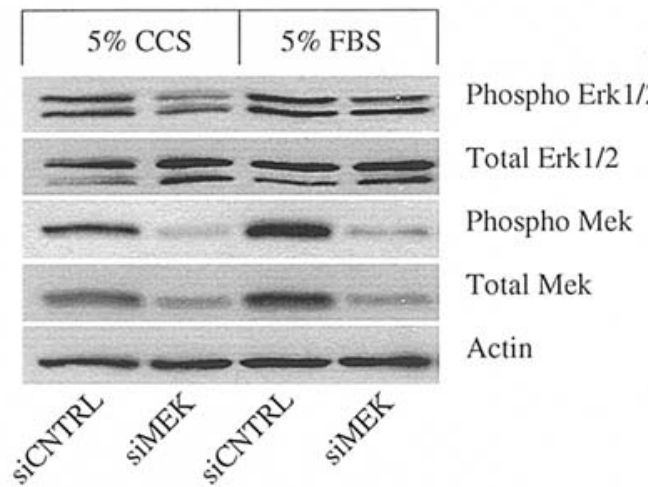

B)
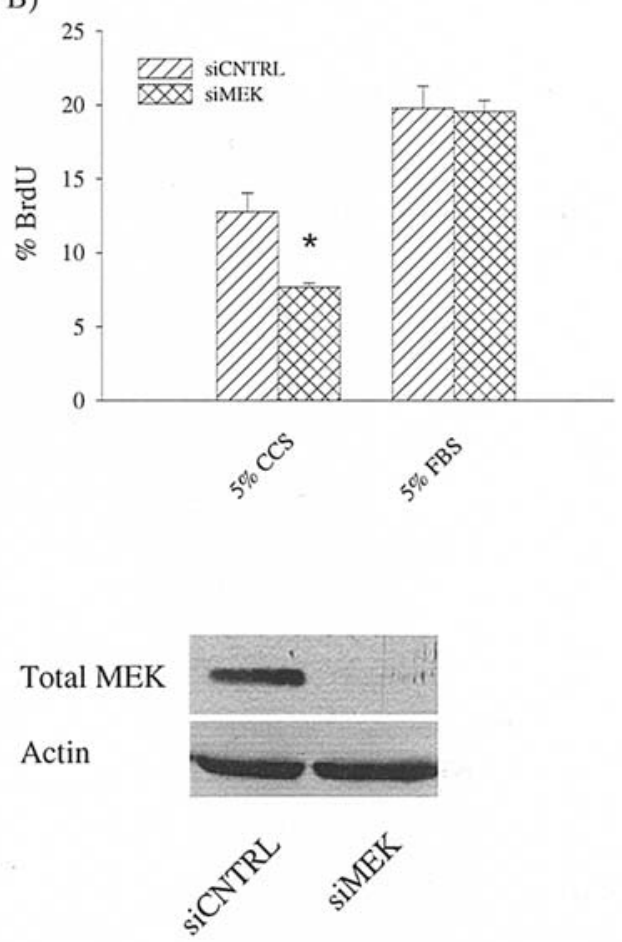

Figure 1. MEK signaling is not essential for fetal bovine serum-induced DNA synthesis of ML-20 breast carcinoma cells. Cells were cotransfected with siRNA duplexes targeting MEK1 and MEK2 or with non-targeting control siRNA. (A) Western blot analysis of phosphorylated and total levels of ERK1/2 and MEK: Cells were stripped in 3 separate changes of 5\% CCS containing medium 24-h post-transfection followed by incubation overnight in plain PRF-IMEM. Twenty-four hours later cells were treated with either $5 \%$ CCS or 5\% FBS for 15 min prior to lysing of cells. (B) BrdU incorporation assays: Cells were stripped as described above and incubated overnight in plain PRF-IMEM. Twenty-four hours later medium was replaced with either PRF-IMEM containing 5\% CCS or PRF-IMEM containing 5\% fetal bovine serum (FBS) and cells were incubated for an additional $24 \mathrm{~h}$. Cells were then pulsed for 30 min with BrdU and harvested for FACS analysis. ${ }^{*} \mathrm{p}<0.05$.

pulsing cells with BrdU. Twenty-four hours after the addition of the conditional media, cells were pulsed for $30 \mathrm{~min}$ with BrdU prior to harvest according to the manufacturer's recommendations (BrdU flow kit, BD Biosciences, San Diego, CA). BrdU incorporation was analyzed by FACS analysis. For each set of BrdU assays, a set of dishes were transfected alongside the BrdU dishes to probe for total MEK protein via Western blot to ensure that MEK remains depleted at the 24-h timepoint.

\section{Results}

Cotransfection of siRNA targeting MEK1 and MEK2 does not inhibit fetal bovine serum-induced BrdU incorporation in ML-20 breast carcinoma cells. We first sought to determine the effects of MEK depletion on FBS-induced ERK1/2 and MEK phosphorylation in ML-20 cells, an estrogen-responsive MCF-7 sub-line derived in our laboratory $(24,25,28)$. Cells were cotransfected with siRNAs targeting MEK1 and MEK2 or with a non-targeting control siRNA and treated for $15 \mathrm{~min}$ in either CCS or FBS. As shown in Fig. 1A, both phosphorylated and total levels of MEK protein were significantly reduced by MEK1/2 siRNA (Fig. 1A) to similar extents in both conditions. However, cotransfection of MEK1/2 siRNAs resulted in only a slight inhibition of ERK1/2 phosphorylation in FBS-treated cells. Phospho-ERK1/2 levels were more clearly reduced in cells incubated in CCS.

We next sought to determine the effects of MEK knockdown on BrdU incorporation in these conditions. As shown in Fig. 1B (upper panel), MEK depletion had no apparent effect on FBS-induced BrdU incorporation in ML-20. This is in contrast to results seen in HCT-116 cells, a colon carcinoma line, where FBS-induced BrdU incorporation was markedly decreased (data not shown). Interestingly, MEK knockdown did result in a marked inhibition of BrdU incorporation in cells incubated in CCS (Fig. 1B, upper panel). Western blot analysis of lysates from cells transfected alongside cells used in the BrdU assays verified that total MEK protein levels were almost completely depleted at the 24-h timepoint after addition of conditions (Fig. 1B, lower panel) suggesting that lack of inhibition of BrdU incorporation in FBS-treated cells was not due to insufficient knockdown.

Cotransfection of siRNA targeting MEK1 and MEK2 does not inhibit 17ß-estradiol-induced BrdU incorporation in ML-20 breast carcinoma cells. The ML-20 cell line is an estrogen responsive cell line and it is possible that the increase in DNA synthesis seen in cells treated with FBS versus cells maintained in $5 \% \mathrm{CCS}$ is due to the $\mathrm{E}_{2}$ component of FBS. To clarify the dependence on MEK for $\mathrm{E}_{2}$-induced DNA synthesis in ML-20 cells, we next sought to determine the effect of MEK knockdown on $\mathrm{E}_{2}$-induced ERK1/2 phosphorylation and BrdU incorporation. Control and MEK transfected ML-20 cells that were stripped of estrogens in CCS containing medium were either placed in fresh CCS or treated with CCS plus $\mathrm{E}_{2}\left(10^{-8} \mathrm{M}\right)$ for $15 \mathrm{~min}$. As shown in Fig. 2A, ERK1/2 phosphorylation is reduced in cells cotransfected with MEK1/2 siRNA versus control transfected cells in CCS or CCS plus $\mathrm{E}_{2}$ containing medium. Both total and activated levels of MEK were almost completely abolished in MEK siRNA transfected cells as compared to control transfected cells (Fig. 2A). Next, control and MEK transfected ML-20 cells were stripped as described above and placed in fresh CCS or treated with CCS plus $\mathrm{E}_{2}$ $\left(10^{-8} \mathrm{M}\right)$ for $24 \mathrm{~h}$ followed by BrdU incorporation and subsequent FACS analysis. As shown in Fig. 2B (upper panel), $\mathrm{E}_{2}$-induced BrdU incorporation in ML-20 cells was 
A)

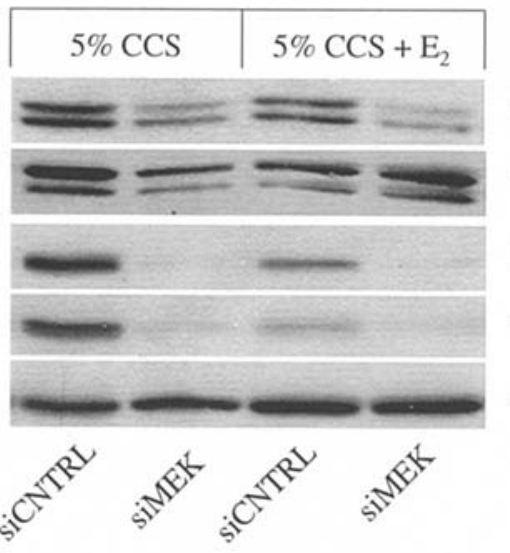

B)

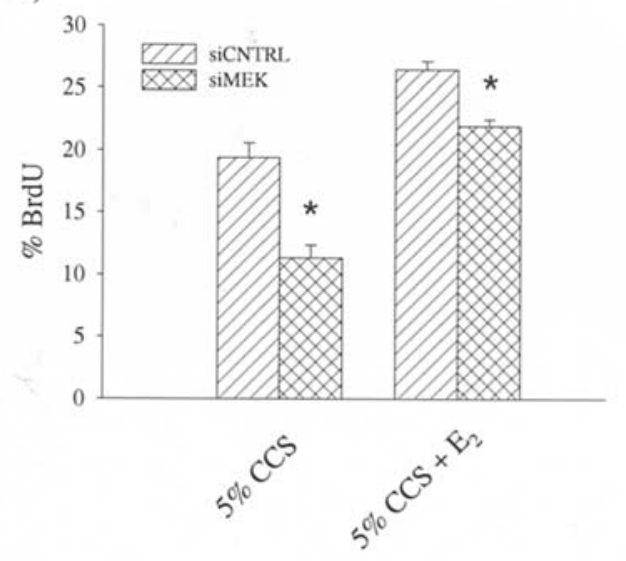

Total Mek

Actin
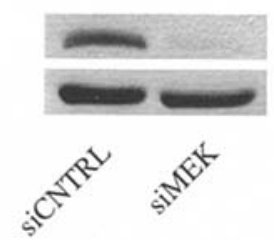

Figure 2. MEK is not essential for $17 ß$-estradiol $\left(\mathrm{E}_{2}\right)$ mediated DNA synthesis of ML-20 breast carcinoma cells. (A) Western blot analysis of phosphorylated and total levels of ERK1/2 and MEK: Cells were stripped as described in Fig. 1 and incubated overnight in plain PRF-IMEM. Twentyfour hours later medium was replaced with either $5 \%$ CCS or $5 \%$ CCS plus $10^{-8} \mathrm{M}$ final concentration $\mathrm{E}_{2}$ for 15 min prior to lysing of cells. (B) BrdU incorporation assays: Cells were transfected and stripped as described in Fig. 1. Twenty-four hours later medium was replaced with either 5\% CCS or $5 \%$ CCS plus $10^{-8} \mathrm{M}$ final concentration $\mathrm{E}_{2}$ and cells were incubated for an additional $24 \mathrm{~h}$ prior to a 30 -min pulse with $\mathrm{BrdU} ;{ }^{*} \mathrm{p}<0.05$.

only moderately inhibited by RNAi mediated knockdown of MEK1 and MEK2 compared to cells transfected with a control siRNA duplex. Lysates were isolated from cells transfected in the same experiment to determine the extent of MEK protein knockdown $24 \mathrm{~h}$ after addition of conditional media in the BrdU dishes. As shown in Fig. 2B (lower panel), MEK protein in these cells was nearly undetectable.
A)

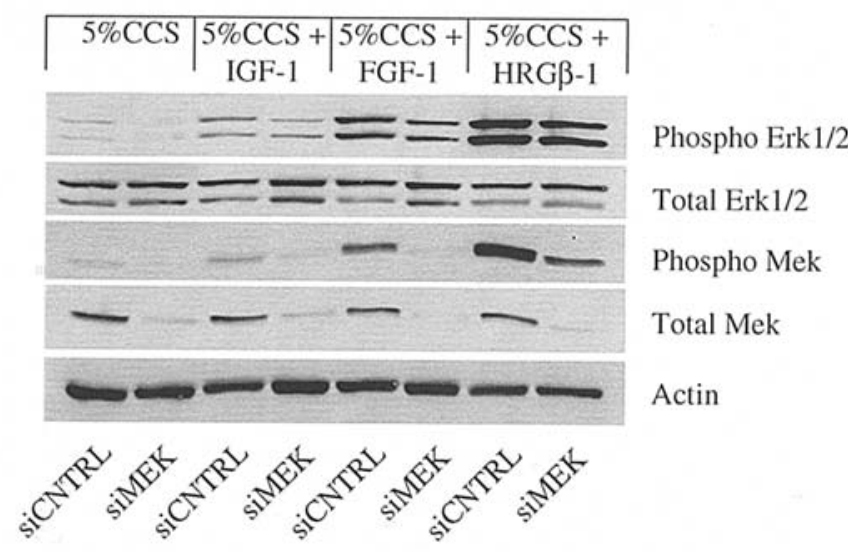

B)

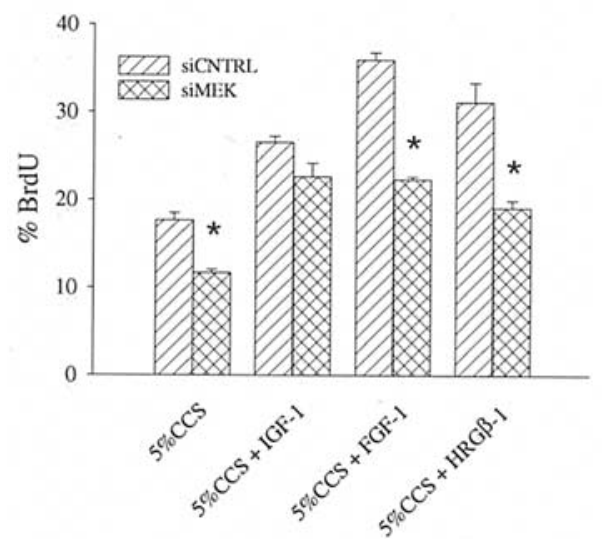

Total Mek

Actin

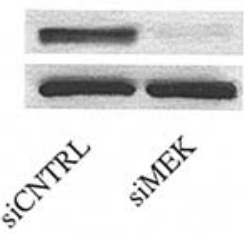

Figure 3. FGF1 or HRGß1-induced BrdU incorporation is repressed by MEK siRNA treatment but remains elevated compared to control cells. (A) Western blot analysis of phosphorylated and total levels of ERK1/2 and MEK: Cells were stripped as described in Fig. 1 and incubated overnight in plain IMEM. Twenty-four hours later cells were treated with IGF-1, FGF-1, or HRGß1 in 5\% CCS at the concentrations listed below for 15 min prior to lysing of cells. (B) BrdU incorporation assays: Cells were transfected and stripped as described above. Twenty-four hours later medium was replaced with either 5\% CCS or 5\% CCS plus IGF-1 (20 ng/ml), FGF-1 $(20 \mathrm{ng} / \mathrm{ml})$, or HRGß1 $(50 \mathrm{ng} / \mathrm{ml})$ and cells were incubated for an additional $24 \mathrm{~h}$ prior to a 30-min pulse with BrdU; ${ }^{*} \mathrm{p}<0.05$.

Growth factor-induced BrdU incorporation in $M L-20$ breast carcinoma cells is differentially affected by cotransfection of MEK1 and MEK2 siRNA in charcoal-stripped serum. We next explored the ability of specific growth factors to induce DNA synthesis and to test the effect of MEK1/2 knockdown on growth factor-induced ERK1/2 phosphorylation and DNA synthesis in ML-20 cells. We first determined the effects of 
A)

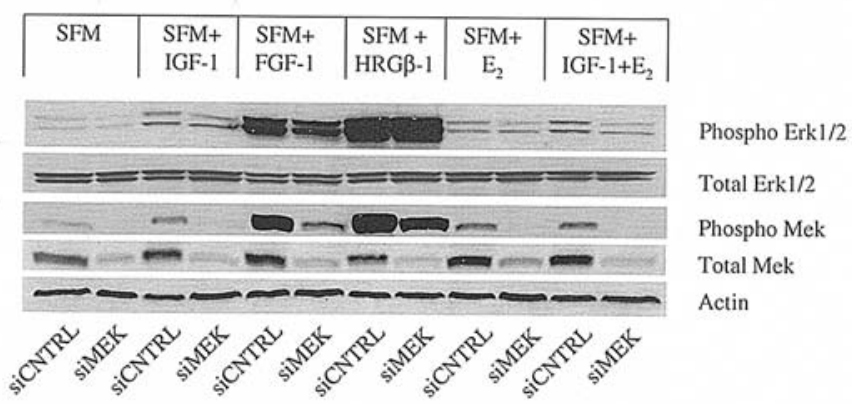

B)

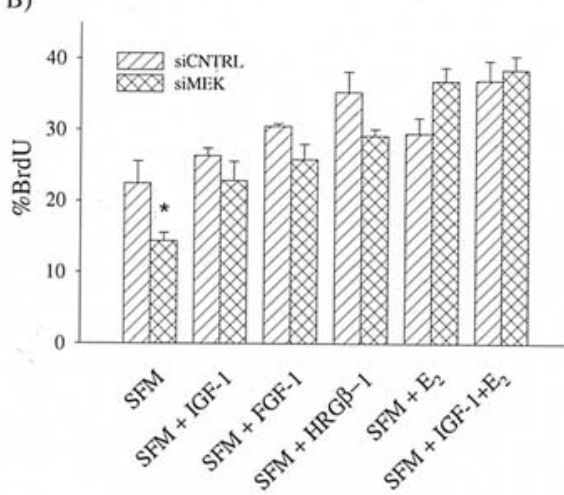

Total Mek

Actin

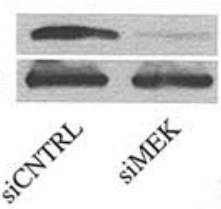

Figure 4. MEK inhibition does not markedly affect growth factor or $\mathrm{E}_{2}$ induced DNA synthesis in serum-free medium. (A) Western blot analysis of phosphorylated and total levels of ERK1/2 and MEK: Cells were stripped as described in Fig. 1 and incubated overnight in plain IMEM. Twenty-four hours later cells were treated with IGF-1, FGF-1, or HRGß1 at the concentrations listed above for 15 min prior to lysing of cells. (B) BrdU incorporation assays: Cells were transfected and stripped as described above. Twenty-four hours later medium was replaced with either serum-free medium or serum-free medium plus IGF-1 $(20 \mathrm{ng} / \mathrm{ml})$, FGF-1 $(20 \mathrm{ng} / \mathrm{ml})$, HRGß1 (50 ng/ml), $\mathrm{E}_{2}\left(10^{-8} \mathrm{M}\right)$, or IGF-1 $(20 \mathrm{ng} / \mathrm{ml})+\mathrm{E}_{2}\left(10^{-8} \mathrm{M}\right)$ and cells were incubated for an additional $24 \mathrm{~h}$ prior to a 30 -min pulse with BrdU; ${ }^{*} \mathrm{p}<0.01$.

MEK knockdown on IGF-1-, FGF-1-, or HRGß1-induced ERK1/2 phosphorylation in lysates isolated from cells cotransfected with MEK1 and MEK2 siRNA and treated with these factors for $15 \mathrm{~min}$ in CCS containing medium. MEK depletion led to minimal reduction in phosphorylated ERK1/2 for all three treatment groups relative to control transfected cells although both phospho and total MEK levels are clearly reduced (Fig. 3A). Next, we determined the effects of MEK ablation on DNA synthesis in cells transfected as described above and treated with growth factors in CCS-containing medium for $24 \mathrm{~h}$ using BrdU incorporation assays. Transfection of cells with siRNA targeting MEK1/2 clearly inhibited FGF-1- and HRGß1-induced BrdU incorporation compared to control transfected cells but did not bring it down to basal levels. In contrast, MEK1/2 knockdown had no significant effect on IGF-1-induced DNA synthesis (Fig. 3B, upper panel). As in previous assays, cells that were maintained in CCS also showed a reduction in BrdU incorporation in MEK1/2 transfected cells versus control transfected cells (Fig. 3B, upper panel). Western analysis of cells transfected in parallel to cells used in the BrdU assays showed that total MEK protein was reduced to barely detectable levels at the 24-h timepoint (Fig. 3B, lower panel).

Cotransfection of siRNA targeting MEK1 and MEK2 does not significantly inhibit IGF-1-, FGF-1-, or HRGß1-induced BrdU incorporation in serum-free medium. Due to the heterogeneous nature of serum, we could not rule out that any apparent effects of MEK knockdown on DNA synthesis seen in cells maintained and treated in CCS containing media were due to inhibition of mechanisms related to serum components and not signaling mechanisms activated by the exogenous growth factors. Therefore, we further tested the effects of MEK depletion on growth factor and $\mathrm{E}_{2}$-induced ERK1/2 activation and BrdU incorporation in serum-free medium. Previous studies by Sutherland and co-investigators (30) have shown that $E_{2}$ in the absence of serum is not a very potent inducer of DNA synthesis in MCF-7 cells, but that it can synergize with IGF-1 in this condition and induce DNA synthesis. Therefore, we also tested the effect of MEK depletion on cells treated with $\mathrm{E}_{2}$ plus IGF-1 in SFM. As shown in Fig. 4A, knockdown of MEK does not lead to a significant inhibition of ERK1/2 phosphorylation induced by the growth factors, $\mathrm{E}_{2}$, or a combination of $\mathrm{E}_{2}$ plus IGF-1, despite a clear reduction in both total and phospho MEK levels. We next treated cells as described above in SFM and observed for effects of MEK1/2 inhibition on DNA synthesis. As shown in Fig. 4B (upper panel), MEK knockdown showed minimal or no effect on growth factor, $\mathrm{E}_{2}$, or $\mathrm{E}_{2}$ plus IGF-1-induced DNA synthesis in SFM compared to non-treated cells. Background levels of BrdU incorporation were reduced by MEK knockdown in SFM indicating that MEK siRNA were functional (Fig. 4B, upper panel). Western blot analysis of cells transfected in parallel with those used in the BrdU assays showed that total MEK levels in these cells were reduced to nearly undetectable levels (Fig. 4B, lower panel).

\section{Discussion}

Deregulation of the Ras-Raf-MEK-ERK signaling pathway has been shown to play a role in the development and progression of many types of cancer. In breast carcinoma cells, aberrant MEK signaling has been linked to proliferation, migration, angiogenesis, and the development of resistance to antiestrogens (23,31-33). Despite this, the role of MEK signaling in ER-positive breast carcinoma cells, particularly in regards to the activation of ERK1/2 by $E_{2}$ and the effects of serum on $E_{2}$ or growth factor-mediated signaling, remains unclear.

In this study, we show that RNAi-mediated knockdown of MEK had no significant effect on BrdU incorporation in 
ML-20 cells maintained in FBS although there was a clear reduction in DNA synthesis in cells maintained in CCS (Fig. 1B). Western blot analysis of total MEK protein levels from cells transfected in parallel dishes indicates that the lack of effect seen on FBS-induced BrdU incorporation in ML-20 cells is not due to inefficient knockdown of MEK (Fig. 1B). Together, these results suggest that FBS-induced DNA synthesis is insensitive to MEK depletion in ML-20 cells or that the remaining levels of activated MEK are sufficient to drive cell cycle progression. This is not surprising in light of studies showing that MCF-7 cells maintained in serum containing medium were refractory to the effects of the MEK inhibitor PD184352 in their ability to form colonies in soft agar (14). Western blot analysis of ERK1/2 and MEK in cells maintained in FBS-containing medium showed that there was only a slight inhibition of FBS-induced ERK1/2 phosphorylation in contrast to a clear reduction in phosphoERK1/2 levels in cells maintained in CCS (Fig. 1A). Both total and phosphorylated MEK levels were markedly reduced under both conditions. These results suggest that serum components other than $\mathrm{E}_{2}$ are at least partially involved in driving DNA synthesis and that the phosphorylation of the downstream ERKs by these components is sensitive to loss of MEK activity, as is the increased DNA synthesis mediated by these components.

To further explore the possible role of MEK-mediated DNA synthesis in $\mathrm{E}_{2}$-induced growth we next assessed the effects of MEK knockdown on $\mathrm{E}_{2}$-induced DNA synthesis. MEK knockdown resulted in only a modest reduction in DNA synthesis in $\mathrm{E}_{2}$-treated cells maintained in CCScontaining medium compared to control transfected cells despite a clear inhibition of total MEK protein (Fig. 2B). It is important to note that the level of BrdU incorporation observed in CCS plus $E_{2}$ in cells cotransfected with MEK siRNAs remain elevated compared to CCS alone. This indicates that MEK independent pathways are contributing to $\mathrm{E}_{2}$-induced DNA synthesis and suggests that the minimal reduction observed is related to effects of MEK siRNAs on signaling via serum components. $\mathrm{E}_{2}$ did not further increase phosphorylation of ERK1/2 over that induced by CCS in our assays despite resulting in increased BrdU incorporation (Fig. 2A). This is not entirely surprising as other studies have suggested that $\mathrm{E}_{2}$ can be mitogenic without detectable activation of ERK1/2 in MCF-7 cells $(19,21)$.

Several studies have suggested that serum components can significantly influence the outcome of studies using ERpositive MCF-7 cells. In a recent report aimed at clarifying the conflicting data that exist concerning $\mathrm{E}_{2}$-induced ERK1/2 phosphorylation, Brower et al showed that $\mathrm{E}_{2}$ administered to MCF-7 cells in the presence of charcoal-stripped serum induced ERK1/2 phosphorylation but $\mathrm{E}_{2}$ administered in SFM resulted in no significant change in ERK1/2 phosphorylation levels and attributed the effects seen in the presence of serum on other factors present in the serum (34). These results are in contrast to our results showing that there was no increase in ERK1/2 phosphorylation in response to $\mathrm{E}_{2}$ in CCS-containing media (Fig. 2). Other studies have also reported no significant increase in ERK1/2 phosphorylation in response to $\mathrm{E}_{2}(22,35,36)$.
To clarify the role of MEK signaling in DNA synthesis induced by growth factors in ML-20 cells, we looked at the effects of MEK knockdown on DNA synthesis induced by three separate growth factors that have been shown to be mitogenic in MCF-7 cells. ML-20 cells treated with IGF-1, FGF-1 or HRGß1 in CCS-containing medium showed an increase in ERK1/2 phosphorylation and DNA synthesis relative to non-treated control cells (Fig. 3A and B). DNA synthesis induced by IGF-1 was not significantly inhibited by knockdown of MEK whereas that of FGF-1- and HRGß1treated cells was moderately reduced (Fig. 3B). MCF-7 cells treated with IGF-1 plus $\mathrm{E}_{2}$, which has been shown to increase DNA synthesis in serum-free media to a greater degree than either stimulus alone (30), resulted in a relative increase in BrdU incorporation compared to either treatment alone that was not lost in cells cotransfected with MEK siRNAs. These results are in agreement with previous studies in which inhibition of the MAPK signaling pathway by PD98059 did not inhibit IGF-1 induced stimulation of cyclin D1 synthesis, hyperphosphorylation of the retinoblastoma protein $\mathrm{pRb}$, DNA synthesis, and cell division in MCF-7 cells (37). These same studies indicated a potential role for phosphatidylinositol 3-kinase (PI3-K) in IGF-1-induced cell signaling as the PI3-K inhibitor LY294002 did inhibit the observed stimulatory effects of IGF-1 in these cells. It is possible therefore that the inability of MEK knockdown to inhibit IGF-1-induced DNA synthesis in our assays is due to activation of additional signaling pathways such as the PI3-K signaling pathway.

Alternatively, the effects of MEK knockdown seen on DNA synthesis induced by FGF-1 or HRGß1 in CCS-containing medium could be due to effects on DNA synthesis induced by serum components other than these growth factors. Additional studies have suggested that serum components that are not affected by dextran-charcoal treatment, such as sex hormone binding globulin and bovine serum albumin, can have a significant impact on the ability of $\mathrm{E}_{2}$ to exert mitogenic effects in MCF-7 cells (38-40). It is possible that these factors could also influence the stimulatory effects of FGF-1 or HRGß1. We show that the inhibition of DNA synthesis induced by growth factors in the presence of stripped serum is not significantly reduced when the same growth factors are used in SFM (Fig. 4B). This suggests that serum components at least partially influence MEK-mediated DNA synthesis induced by these growth factors and that DNA synthesis induced by IGF-1, FGF-1, HRGß1, or by $\mathrm{E}_{2}$ is at least partially independent of MEK activity.

Our results also suggest that ERK1/2 activation by growth factors is unaffected by MEK ablation. These results are consistent with our previous observations that showed that the sustained, high magnitude ERK activation in ML-20 cells induced by FGF-1 or HRGß1 is less susceptible to the MEK activation inhibitors U0126, PD98059, and PD184161 (27). In the intracellular environment MEK is known to interact with various binding proteins and to be involved in complexes with additional signaling molecules and scaffolding proteins (41-43). Scaffold proteins not only promote simultaneous interaction of MEK with its substrate ERKs, but also allosterically regulate these kinases (44). Through such interactions, constitutive levels of MEK could be more important in signaling complexes that are integral to serum-specific ERK1/2 
activation, but less important to growth factor-specific ERK1/2 activation pathways. Therefore, inactivation or reduction of MEK could affect serum stimulated ERK1/2 activation, without affecting that stimulated by growth factors, thus explaining the stimulus-specific susceptibility to MEK ablation.

Taken together, these results suggest that serum components other than estrogen that mediate DNA synthesis in MCF-7 cells are sensitive to MEK depletion. In contrast, DNA synthesis induced by $\mathrm{E}_{2}$, IGF-1, FGF-1, or HRGß1 in $\mathrm{MCF}-7$ cells is at least partially independent of MEK signaling since MEK depletion did not reduce BrdU incorporation to basal levels. The modest reduction that is observed in growth factor or $\mathrm{E}_{2}$ containing medium with MEK knockdown may be due in part to abrogation of MEK-dependent signaling mediated by serum components that are present after treatment with dextran-coated charcoal. These findings suggest that the potential effects of whole or stripped serum should be taken into account when observing effects of exogenous treatments in ER-positive MCF-7 breast cancer cell lines.

\section{Acknowledgements}

The work was supported by the Department of Defense Breast Cancer Training grant DAMD 17-01-1-0400 (N.R.E.) and NIH grant CA50376 (F.G.K.); UAB Comprehensive Cancer Center Core grant P30 CA13148. This work was also supported by the Adolph Weil Endowed Chair in Cancer Biology at Southern Research Institute (F.G.K.). J.V.T. is the recipient of a Susan G. Komen Breast Cancer Foundation award.

\section{References}

1. Dent P, Haser W, Haystead TAJ, Vincent LA, Roberts TM and Sturgill TW: Activaiton of mitogen-activated protein kinase kinase by v-raf in NIH 3 T3 cells and in vitro. Science 257: 1404-1407, 1992.

2. Lester RD, Jo M, Campana WM and Gonias SL: Erythropoietin promotes MCF-7 breast cancer cell migration by a ERK/MAP kinase-dependent pathway and is primarily responsible for the increase in migration observed in hypoxia. J Biol Chem 280: 39273-39277, 2005.

3. Jung JW, Cho SD, Ahn NS, et al: Ras/MAP kinase pathways are involved in Ras specific apoptosis induced by sodium butyrate. Cancer Lett 225: 199-206, 2005.

4. Chang X, Tou JC, Hong C, et al: 3,3'-Diindolylmethane inhibits angiogenesis and the growth of transplantable human breast carcinoma in athymic mice. Carcinogenesis 26: 771-778, 2005.

5. Ostrakhovitch EA and Cherian MG: Inhibition of extracellular signal regulated kinase (ERK) leads to apoptosis inducing factor (AIF) mediated apoptosis in epithelial breast cancer cells: the lack of effect of ERK in p53 mediated copper induced apoptosis. J Cell Biochem 95: 1120-1134, 2005.

6. El-Ashry D, Miller DL, Kharbanda S, Lippman ME and Kern FG: Constitutive Raf-1 kinase activity in breast cancer cells induces both estrogen-independent growth and apoptosis. Oncogene 15: 423-435, 1997.

7. Donovan JC, Milic A and Slingerland JM: Constitutive MEK/ MAPK activation leads to p27(Kip1) deregulation and antiestrogen resistance in human breast cancer cells. J Biol Chem 276: 40888-40895, 2001.

8. Coutts AS and Murphy LC: Elevated mitogen-activated protein kinase activity in estrogen-nonresponsive human breast cancer cells. Cancer Res 58: 4071-4074, 1998.

9. Oh AS, Lorant LA, Holloway JN, Miller DL, Kern FG and El-Ashry D: Hyperactivation of MAPK induces loss of ERalpha expression in breast cancer cells. Mol Endocrinol 15: 1344-1359, 2001.
10. Castoria G, Barone MV, Di DM, et al: Non-transcriptional action of oestradiol and progestin triggers DNA synthesis. EMBO J 18: 2500-2510, 1999.

11. Dupont J, Karas M and LeRoith D: The cyclin-dependent kinase inhibitor $\mathrm{p} 21 \mathrm{CIP} / \mathrm{WAF}$ is a positive regulator of insulin-like growth factor I-induced cell proliferation in MCF-7 human breast cancer cells. J Biol Chem 278: 37256-37264, 2003.

12. Weng LP, Smith WM, Brown JL and Eng C: PTEN inhibits insulin-stimulated MEK/MAPK activation and cell growth by blocking IRS-1 phosphorylation and IRS-1/Grb-2/Sos complex formation in a breast cancer model. Hum Mol Genet 10: 605-616, 2001 .

13. Ripple MO, Kalmadi S and Eastman A: Inhibition of either phosphatidylinositol 3-kinase/Akt or the mitogen/extracellularregulated kinase, MEK/ERK, signaling pathways suppress growth of breast cancer cell lines, but MEK/ERK signaling is critical for cell survival. Breast Cancer Res Treat 93: 177-188, 2005 .

14. Sebolt-Leopold JS, Dudley DT, Herrera R, et al: Blockade of the MAP kinase pathway suppresses growth of colon tumors in vivo. Nat Med 5: 810-816, 1999.

15. Weldon CB, McKee A, Collins-Burow BM, et al: PKC-mediated survival signaling in breast carcinoma cells: a role for MEK1AP1 signaling. Int J Oncol 26: 763-768, 2005.

16. Gilad LA, Bresler T, Gnainsky J, Smirnoff P and Schwartz B: Regulation of vitamin D receptor expression via estrogeninduced activation of the ERK $1 / 2$ signaling pathway in colon and breast cancer cells. J Endocrinol 185: 577-592, 2005.

17. Razandi M, Pedram A, Rosen EM and Levin ER: BRCA1 inhibits membrane estrogen and growth factor receptor signaling to cell proliferation in breast cancer. Mol Cell Biol 24: 5900-5913, 2004.

18. Castoria G, Migliaccio A, Bilancio A, et al: PI3-kinase in concert with Src promotes the S-phase entry of oestradiol-stimulated MCF-7 cells. EMBO J 20: 6050-6059, 2001.

19. Lobenhofer EK, Huper G, Iglehart JD and Marks JR: Inhibition of mitogen-activated protein kinase and phosphatidylinositol 3kinase activity in MCF-7 cells prevents estrogen-induced mitogenesis. Cell Growth Differ 11: 99-110, 2000.

20. Lewis JS, Vijayanathan V, Thomas TJ, et al: Activation of cyclin D1 by estradiol and spermine in MCF-7 breast cancer cells: a mechanism involving the p38 MAP kinase and phosphorylation of ATF-2. Oncol Res 15: 113-128, 2005.

21. Gaben AM, Saucier C, Bedin M, Redeuilh G and Mester J: Mitogenic activity of estrogens in human breast cancer cells does not rely on direct induction of mitogen-activated protein kinase/extracellularly regulated kinase or phosphatidylinositol 3-kinase. Mol Endocrinol 18: 2700-2713, 2004.

22. Bonapace IM, Addeo R, Altucci L, et al: 17 beta-estradiol overcomes a G1 block induced by $\mathrm{HMG}-\mathrm{CoA}$ reductase inhibitors and fosters cell cycle progression without inducing ERK-1 and -2 MAP kinases activation. Oncogene 12: 753-763, 1996.

23. McLeskey SW, Kurebayashi J, Honig SF, et al: Fibroblast growth factor 4 transfection of MCF-7 cells produces cell lines that are tumorigenic and metastatic in ovariectomized or tamoxifen-treated athymic nude mice. Cancer Res 53: 2168-2177, 1993.

24. McLeskey SW, Zhang L, Kharbanda S, et al: Fibroblast growth factor overexpressing breast carcinoma cells as models of angiogenesis and metastasis. Breast Cancer Res Treat 39: 103-117, 1996.

25. Zhang L, Kharbanda S, Chen D, et al: MCF-7 breast carcinoma cells overexpressing FGF-1 form vascularized, metastatic tumors in ovariectomized or tamoxifen-treated nude mice. Oncogene 15: 2093-2108, 1997.

26. Zhang L, Kharbanda S, Hanfelt J and Kern FG: Both autocrine and paracrine effects of transfected acidic fibroblast growth factor are involved in the estrogen-independent and antiestrogenresistant growth of MCF-7 breast cancer cells. Cancer Res 58: 352-361, 1998.

27. Thottassery JV, Sun Y, Westbrook L, et al: Prolonged extracellular signal-regulated kinase $1 / 2$ activation during fibroblast growth factor 1 - or heregulin beta1-induced antiestrogenresistant growth of breast cancer cells is resistant to mitogenactivated protein/extracellular regulated kinase kinase inhibitors. Cancer Res 64: 4637-4647, 2004.

28. McLeskey SW, Zhang L, El-Ashry D, et al: Tamoxifen-resistant FGF-transfected MCF-7 cells are cross-resistant in vivo to the antiestrogen, ICI 182,780 and two aromatase inhibitors. Clin Cancer Res 4: 697-711, 1998. 
29. Ussar S and Voss T: MEK1 and MEK2, different regulators of the G1/S transition. J Biol Chem 279: 43861-43869, 2004.

30. Lai A, Sarcevic B, Prall OW and Sutherland RL: Insulin/insulinlike growth factor-I and estrogen cooperate to stimulate cyclin E-Cdk2 activation and cell cycle progression in MCF-7 breast cancer cells through differential regulation of cyclin $\mathrm{E}$ and p21WAF1/Cip1. J Biol Chem 276: 25823-25833, 2001.

31. Nguyen DH, Webb DJ, Catling AD, et al: Urokinase-type plasminogen activator stimulates the Ras/Extracellular signalregulated kinase (ERK) signaling pathway and MCF-7 cell migration by a mechanism that requires focal adhesion kinase, Src, and Shc. Rapid dissociation of GRB2/Sps-Shc complex is associated with the transient phosphorylation of ERK in urokinase-treated cells. J Biol Chem 275: 19382-19388, 2000.

32. Eliceiri BP, Klemke R, Stromblad S and Cheresh DA: Integrin alphavbeta3 requirement for sustained mitogen-activated protein kinase activity during angiogenesis. J Cell Biol 140: 1255-1263, 1998.

33. Arteaga CL, Carty-Dugger T, Moses HL, Hurd SD and Pietenpol JA: Transforming growth factor beta 1 can induce estrogen-independent tumorigenicity of human breast cancer cells in athymic mice. Cell Growth Differ 4: 193-201, 1993.

34. Brower SL, Roberts JR, Antonini JM and Miller MR: Difficulty demonstrating estradiol-mediated ERK1/2 phosphorylation in MCF-7 cells. J Steroid Biochem Mol Biol 96: 375-385, 2005.

35. Caristi S, Galera JL, Matarese F, et al: Estrogens do not modify MAP kinase-dependent nuclear signaling during stimulation of early $\mathrm{G}(1)$ progression in human breast cancer cells. Cancer Res 61: 6360-6366, 2001.

36. Joel PB, Traish AM and Lannigan DA: Estradiol-induced phosphorylation of serine 118 in the estrogen receptor is independent of p42/p44 mitogen-activated protein kinase. J Biol Chem 273: 13317-13323, 1998.
37. Dufourny B, Alblas J, van Teeffelen HA, et al: Mitogenic signaling of insulin-like growth factor I in MCF-7 human breast cancer cells requires phosphatidylinositol 3-kinase and is independent of mitogen-activated protein kinase. J Biol Chem 272: 31163-31171, 1997.

38. Fortunati N, Fissore F, Fazzari A, et al: Estradiol induction of cAMP in breast cancer cells is mediated by foetal calf serum (FCS) and sex hormone-binding globulin (SHBG). J Steroid Biochem Mol Biol 70: 73-80, 1999.

39. Bradlow HL, Arcuri F, Blasi L and Castagnetta L: Effect of serum albumin on estrogen metabolism in human cancer cell lines. Mol Cell Endocrinol 115: 221-225, 1995.

40. Geier A, Beery R, Haimshon M, Hemi R and Lunenfeld B: Serum and insulin inhibit cell death induced by cycloheximide in the human breast cancer cell line MCF-7. In Vitro Cell Dev Biol 28A: 415-418, 1992.

41. Nguyen A, Burack WR, Stock JL, et al: Kinase suppressor of Ras (KSR) is a scaffold which facilitates mitogen-activated protein kinase activation in vivo. Mol Cell Biol 22: 3035-3045, 2002.

42. Sharma C, Vomastek T, Tarcsafalvi A, et al: MEK partner 1 (MP1): regulation of oligomerization in MAP kinase signaling. J Cell Biochem 94: 708-719, 2005.

43. Pullikuth A, McKinnon E, Schaeffer HJ and Catling AD: The MEK1 scaffolding protein MP1 regulates cell spreading by integrating PAK1 and Rho signals. Mol Cell Biol 25: 5119-5133, 2005.

44. Dard N and Peter M: Scaffold proteins in MAP kinase signaling: more than simple passive activating platforms. Bioessays 28: 146-156, 2006. 